\title{
Combined Analysis of Neutrino and Antineutrino Oscillations at T2K
}

K. Abe, ${ }^{47}$ J. Amey, ${ }^{16}$ C. Andreopoulos,${ }^{45,26}$ M. Antonova, ${ }^{21}$ S. Aoki, ${ }^{23}$ A. Ariga, ${ }^{1}$ D. Autiero, ${ }^{28}$ S. Ban, ${ }^{24}$ M. Barbi, ${ }^{39}$ G. J. Barker, ${ }^{55}$ G. Barr,${ }^{35}$ C. Barry, ${ }^{26}$ P. Bartet-Friburg, ${ }^{36}$ M. Batkiewicz, ${ }^{12}$ V. Berardi, ${ }^{17}$ S. Berkman,,${ }^{3,51}$ S. Bhadra, ${ }^{60}$ S. Bienstock, ${ }^{36}$ A. Blondel, ${ }^{11}$ S. Bolognesi,${ }^{5}$ S. Bordoni, ${ }^{14}$ S. B. Boyd ${ }^{55}$ D. Brailsford ${ }^{25}$ A. Bravar,${ }^{11}$ C. Bronner,${ }^{22}$ M. Buizza Avanzini, ${ }^{9}$ R. G. Calland, ${ }^{22}$ T. Campbell, ${ }^{7}$ S. Cao, ${ }^{13}$ S. L. Cartwright, ${ }^{43}$ M. G. Catanesi, ${ }^{17}$ A. Cervera, ${ }^{15}$ C. Checchia, ${ }^{19}$ D. Cherdack, ${ }^{7}$ N. Chikuma, ${ }^{46}$ G. Christodoulou, ${ }^{26}$ A. Clifton, ${ }^{7}$ J. Coleman ${ }^{26}$ G. Collazuol, ${ }^{19}$ D. Coplowe, ${ }^{35}$ A. Cudd, ${ }^{29}$ A. Dabrowska, ${ }^{12}$ G. De Rosa, ${ }^{18}$ T. Dealtry, ${ }^{25}$ P. F. Denner, ${ }^{55}$ S. R. Dennis, ${ }^{26}$ C. Densham, ${ }^{45}$ D. Dewhurst,${ }^{35}$ F. Di Lodovico, ${ }^{38}$ S. Di Luise, ${ }^{10}$ S. Dolan, ${ }^{35}$ O. Drapier, ${ }^{9}$ K. E. Duffy, ${ }^{35}$ J. Dumarchez ${ }^{36}$ M. Dziewiecki, ${ }^{54}$

S. Emery-Schrenk, ${ }^{5}$ A. Ereditato, ${ }^{1}$ T. Feusels, ${ }^{3,51}$ A. J. Finch, ${ }^{25}$ G. A. Fiorentini, ${ }^{60}$ M. Friend, ${ }^{13,}{ }^{*}$ Y. Fujii, ${ }^{13,{ }^{*}}$ D. Fukuda, ${ }^{33}$ Y. Fukuda,${ }^{30}$ V. Galymov, ${ }^{28}$ A. Garcia,${ }^{14}$ C. Giganti, ${ }^{36}$ F. Gizzarelli, ${ }^{5}$ T. Golan,${ }^{58}$ M. Gonin, ${ }^{9}$ D. R. Hadley,${ }^{55}$ L. Haegel,${ }^{11}$ M. D. Haigh, ${ }^{55}$ D. Hansen, ${ }^{37}$ J. Harada, ${ }^{34}$ M. Hartz, ${ }^{22,51}$ T. Hasegawa, ${ }^{13,}{ }^{*}$ N. C. Hastings, ${ }^{39}$ T. Hayashino,${ }^{24}$ Y. Hayato, ${ }^{47,22}$ R. L. Helmer, ${ }^{51}$ A. Hillairet, ${ }^{52}$ T. Hiraki, ${ }^{24}$ A. Hiramoto, ${ }^{24}$ S. Hirota, ${ }^{24}$ M. Hogan, ${ }^{7}$ J. Holeczek,${ }^{44}$ F. Hosomi, ${ }^{46}$ K. Huang,${ }^{24}$ A. K. Ichikawa, ${ }^{24}$ M. Ikeda, ${ }^{47}$ J. Imber, ${ }^{9}$ J. Insler, ${ }^{27}$ R. A. Intonti, ${ }^{17}$ T. Ishida, ${ }^{13,}{ }^{*}$ T. Ishii, ${ }^{13,}{ }^{*}$ E. Iwai, ${ }^{13}$ K. Iwamoto, ${ }^{40}$ A. Izmaylov, ${ }^{15,21}$ B. Jamieson, ${ }^{57}$ M. Jiang, ${ }^{24}$ S. Johnson, ${ }^{6}$ P. Jonsson, ${ }^{16}$ C. K. Jung, ${ }^{32, \dagger}$ M. Kabirnezhad, ${ }^{31}$ A. C. Kaboth, ${ }^{41,45}$ T. Kajita ${ }^{48, \dagger}$ H. Kakuno, ${ }^{49}$ J. Kameda,${ }^{47}$ D. Karlen,${ }^{52,51}$ T. Katori, ${ }^{38}$ E. Kearns, ${ }^{2,22, \dagger}$ M. Khabibullin, ${ }^{21}$ A. Khotjantsev, ${ }^{21}$ H. Kim, ${ }^{34}$ J. Kim, ${ }^{3,51}$ S. King, ${ }^{38}$ J. Kisiel, ${ }^{44}$ A. Knight, ${ }^{55}$ A. Knox, ${ }^{25}$ T. Kobayashi, ${ }^{13,{ }^{*}}$ L. Koch,${ }^{42}$ T. Koga, ${ }^{46}$ A. Konaka, ${ }^{51}$ K. Kondo ${ }^{24}$ L. L. Kormos, ${ }^{25}$ A. Korzenev, ${ }^{11}$ Y. Koshio, ${ }^{33, \dagger}$ K. Kowalik,${ }^{31}$ W. Kropp, ${ }^{4}$ Y. Kudenko, ${ }^{21, \$}$ R. Kurjata, ${ }^{54}$ T. Kutter, ${ }^{27}$ J. Lagoda, ${ }^{31}$ I. Lamont, ${ }^{25}$ M. Lamoureux ${ }^{5}$ E. Larkin, ${ }^{55}$ P. Lasorak,${ }^{38}$ M. Laveder, ${ }^{19}$ M. Lawe, ${ }^{25}$ M. Licciardi, ${ }^{9}$ T. Lindner, ${ }^{51}$ Z. J. Liptak, ${ }^{6}$ R. P. Litchfield ${ }^{16}$ X. Li, ${ }^{32}$ A. Longhin, ${ }^{19}$ J. P. Lopez, ${ }^{6}$ T. Lou, ${ }^{46}$ L. Ludovici, ${ }^{20}$ X. Lu, ${ }^{35}$ L. Magaletti, ${ }^{17}$ K. Mahn, ${ }^{29}$ M. Malek, ${ }^{43}$ S. Manly, ${ }^{40}$ A. D. Marino, ${ }^{6}$ J. F. Martin, ${ }^{50}$ P. Martins, ${ }^{38}$ S. Martynenko, ${ }^{32}$ T. Maruyama, ${ }^{13, *}$ V. Matveev, ${ }^{21}$ K. Mavrokoridis, ${ }^{26}$ W. Y. Ma ${ }^{16}$ E. Mazzucato, ${ }^{5}$ M. McCarthy, ${ }^{60}$ N. McCauley, ${ }^{26}$ K. S. McFarland, ${ }^{40}$ C. McGrew, ${ }^{32}$ A. Mefodiev, ${ }^{21}$ C. Metelko, ${ }^{26}$ M. Mezzetto, ${ }^{19}$ P. Mijakowski, ${ }^{31}$ A. Minamino, ${ }^{59}$ O. Mineev, ${ }^{21}$ S. Mine, ${ }^{4}$ A. Missert, ${ }^{6}$ M. Miura, ${ }^{47, \dagger}$ S. Moriyama, ${ }^{47, \dagger}$ Th. A. Mueller, ${ }^{9}$ J. Myslik, ${ }^{52}$ T. Nakadaira, ${ }^{13, *}$ M. Nakahata, ${ }^{47,22}$ K. G. Nakamura, ${ }^{24}$ K. Nakamura, ${ }^{22,13,{ }^{*}}$ K. D. Nakamura, ${ }^{24}$ Y. Nakanishi, ${ }^{24}$ S. Nakayama, ${ }^{47, \dagger}$ T. Nakaya, ${ }^{24,22}$ K. Nakayoshi, ${ }^{13,{ }^{*}}$ C. Nantais,${ }^{50}$ C. Nielsen, ${ }^{3}$ M. Nirkko, ${ }^{1}$ K. Nishikawa, ${ }^{13, *}$ Y. Nishimura, ${ }^{48}$ P. Novella, ${ }^{15}$ J. Nowak, ${ }^{25}$ H. M. O’Keeffe, ${ }^{25}$ K. Okumura, ${ }^{48,22}$ T. Okusawa, ${ }^{34}$ W. Oryszczak ${ }^{53}$ S. M. Oser, ${ }^{3,51}$ T. Ovsyannikova, ${ }^{21}$ R. A. Owen, ${ }^{38}$ Y. Oyama, ${ }^{13,}$ V. Palladino, ${ }^{18}$ J. L. Palomino, ${ }^{32}$ V. Paolone, ${ }^{37}$ N. D. Patel, ${ }^{24}$ P. Paudyal, ${ }^{26}$ M. Pavin, ${ }^{36}$ D. Payne, ${ }^{26}$ J. D. Perkin, ${ }^{43}$ Y. Petrov,${ }^{3,51}$ L. Pickard,${ }^{43}$ L. Pickering, ${ }^{16}$ E. S. Pinzon Guerra, ${ }^{60}$ C. Pistillo, ${ }^{1}$ B. Popov, ${ }^{36,8}$ M. Posiadala-Zezula, ${ }^{53}$ J.-M. Poutissou, ${ }^{51}$ R. Poutissou ${ }^{51}$ P. Przewlocki, ${ }^{31}$ B. Quilain, ${ }^{24}$ T. Radermacher ${ }^{42}$ E. Radicioni, ${ }^{17}$ P. N. Ratoff, ${ }^{25}$ M. Ravonel, ${ }^{11}$ M. A. Rayner, ${ }^{11}$ A. Redij, ${ }^{1}$ E. Reinherz-Aronis, ${ }^{7}$ C. Riccio, ${ }^{18}$ P. A. Rodrigues,${ }^{40}$ E. Rondio,${ }^{31}$ B. Rossi, ${ }^{18}$ S. Roth, ${ }^{42}$ A. Rubbia, ${ }^{10}$ A. Rychter, ${ }^{54}$ K. Sakashita, ${ }^{13,}{ }^{*}$ F. Sánchez, ${ }^{14}$ E. Scantamburlo ${ }^{11}$ K. Scholberg, ${ }^{8, \dagger}$ J. Schwehr, ${ }^{7}$ M. Scott, ${ }^{51}$ Y. Seiya,${ }^{34}$ T. Sekiguchi, ${ }^{13,{ }^{*}}$ H. Sekiya, ${ }^{47,22, \dagger}$ D. Sgalaberna, ${ }^{11}$ R. Shah, ${ }^{45,35}$ A. Shaikhiev, ${ }^{21}$ F. Shaker, ${ }^{57}$ D. Shaw, ${ }^{25}$ M. Shiozawa, ${ }^{47,22}$ T. Shirahige, ${ }^{33}$ S. Short, ${ }^{38}$ M. Smy, J. T. Sobczyk, ${ }^{48}$ H. Sobel, ${ }^{4,22}$ M. Sorel, ${ }^{15}$ L. Southwell, ${ }^{25}$ J. Steinmann, ${ }^{42}$ T. Stewart, ${ }^{45}$ P. Stowell, ${ }^{43}$ Y. Suda, ${ }^{46}$ S. Suvorov, ${ }^{21}$ A. Suzuki, ${ }^{23}$ S. Y. Suzuki, ${ }^{13, *}$ Y. Suzuki, ${ }^{22}$ R. Tacik, ${ }^{39,51}$ M. Tada, ${ }^{13, *}$ A. Takeda, ${ }^{47}$ Y. Takeuchi, ${ }^{23,22}$ H. K. Tanaka, ${ }^{47,}$ H. A. Tanaka, ${ }^{50,51, \uparrow}$ D. Terhorst,${ }^{42}$ R. Terri, ${ }^{38}$ T. Thakore, ${ }^{27}$ L. F. Thompson, ${ }^{43}$ S. Tobayama, ${ }^{3,51}$ W. Toki, ${ }^{7}$ T. Tomura, ${ }^{47}$ C. Touramanis, ${ }^{26}$ T. Tsukamoto, ${ }^{13, *}$ M. Tzanov, ${ }^{27}$ Y. Uchida, ${ }^{16}$ M. Vagins, ${ }^{22,4}$ Z. Vallari, ${ }^{32}$ G. Vasseur, ${ }^{5}$ T. Vladisavljevic, ${ }^{35,22}$ T. Wachala, ${ }^{12}$ C. W. Walter, ${ }^{8, \dagger}$

D. Wark ${ }^{45,35}$ M. O. Wascko, ${ }^{16,13}$ A. Weber, ${ }^{45,35}$ R. Wendell, ${ }^{24, \dagger}$ R. J. Wilkes, ${ }^{56}$ M. J. Wilking, ${ }^{32}$ C. Wilkinson, ${ }^{1}$

J. R. Wilson, ${ }^{38}$ R. J. Wilson, ${ }^{7}$ C. Wret,${ }^{16}$ Y. Yamada, ${ }^{13, *}$ K. Yamamoto, ${ }^{34}$ M. Yamamoto, ${ }^{24}$ C. Yanagisawa, ${ }^{32, \|}$ T. Yano, ${ }^{23}$ S. Yen, ${ }^{51}$ N. Yershov ${ }^{21}$ M. Yokoyama, ${ }^{46,}$ K. Yoshida, ${ }^{24}$ T. Yuan, ${ }^{6}$ M. Yu ${ }^{60}$ A. Zalewska, ${ }^{12}$ J. Zalipska, ${ }^{31}$ L. Zambelli, ${ }^{13,}{ }^{*}$ K. Zaremba, ${ }^{54}$ M. Ziembicki, ${ }^{54}$ E. D. Zimmerman, ${ }^{6}$ M. Zito, ${ }^{5}$ and J. Żmuda ${ }^{58}$

(T2K Collaboration)

${ }^{1}$ Albert Einstein Center for Fundamental Physics, Laboratory for High Energy Physics (LHEP), University of Bern, Bern, Switzerland

${ }^{2}$ Department of Physics, Boston University, Boston, Massachusetts, USA

${ }^{3}$ Department of Physics and Astronomy, University of British Columbia, Vancouver, British Columbia, Canada

${ }^{4}$ Department of Physics and Astronomy, University of California, Irvine, Irvine, California, USA

${ }^{5} I R F U, C E A$ Saclay, Gif-sur-Yvette, France 
${ }^{6}$ Department of Physics, University of Colorado at Boulder, Boulder, Colorado, USA

${ }^{7}$ Department of Physics, Colorado State University, Fort Collins, Colorado, USA

${ }^{8}$ Department of Physics, Duke University, Durham, North Carolina, USA

${ }^{9}$ Ecole Polytechnique, IN2P3-CNRS, Laboratoire Leprince-Ringuet, Palaiseau, France

${ }^{10}$ Institute for Particle Physics, ETH Zurich, Zurich, Switzerland

${ }^{11}$ Section de Physique, DPNC, University of Geneva, Geneva, Switzerland

${ }^{12}$ H. Niewodniczanski Institute of Nuclear Physics PAN, Cracow, Poland

${ }^{13}$ High Energy Accelerator Research Organization (KEK), Tsukuba, Ibaraki, Japan

${ }^{14}$ Institut de Fisica d'Altes Energies (IFAE), The Barcelona Institute of Science and Technology, Campus UAB, Bellaterra (Barcelona), Spain

${ }^{15}$ IFIC (CSIC and University of Valencia), Valencia, Spain

${ }^{16}$ Department of Physics, Imperial College London, London, United Kingdom

${ }^{17}$ INFN Sezione di Bari and Dipartimento Interuniversitario di Fisica, Università e Politecnico di Bari, Bari, Italy

${ }^{18}$ INFN Sezione di Napoli and Dipartimento di Fisica, Università di Napoli, Napoli, Italy

${ }^{19}$ INFN Sezione di Padova and Dipartimento di Fisica, Università di Padova, Padova, Italy

${ }^{20}$ INFN Sezione di Roma and Università di Roma "La Sapienza," Roma, Italy

${ }^{21}$ Institute for Nuclear Research of the Russian Academy of Sciences, Moscow, Russia

${ }^{22}$ Kavli Institute for the Physics and Mathematics of the Universe (WPI), University of Tokyo Institutes for Advanced Study, University of Tokyo, Kashiwa, Chiba, Japan

${ }^{23}$ Kobe University, Kobe, Japan

${ }^{24}$ Department of Physics, Kyoto University, Kyoto, Japan

${ }^{25}$ Physics Department, Lancaster University, Lancaster, United Kingdom

${ }^{26}$ Department of Physics, University of Liverpool, Liverpool, United Kingdom

${ }^{27}$ Department of Physics and Astronomy, Louisiana State University, Baton Rouge, Louisiana, USA

${ }^{28}$ Université de Lyon, Université Claude Bernard Lyon 1, IPN Lyon (IN2P3), Villeurbanne, France

${ }^{29}$ Department of Physics and Astronomy, Michigan State University, East Lansing, Michigan, USA

${ }^{30}$ Department of Physics, Miyagi University of Education, Sendai, Japan

${ }^{31}$ National Centre for Nuclear Research, Warsaw, Poland

${ }^{32}$ Department of Physics and Astronomy, State University of New York at Stony Brook, Stony Brook, New York, USA

${ }^{33}$ Department of Physics, Okayama University, Okayama, Japan

${ }^{34}$ Department of Physics, Osaka City University, Osaka, Japan

${ }^{35}$ Department of Physics, Oxford University, Oxford, United Kingdom

${ }^{36}$ Laboratoire de Physique Nucléaire et de Hautes Energies (LPNHE), UPMC, Université Paris Diderot, CNRS/IN2P3, Paris, France

${ }^{37}$ Department of Physics and Astronomy, University of Pittsburgh, Pittsburgh, Pennsylvania, USA

${ }^{38}$ School of Physics and Astronomy, Queen Mary University of London, London, United Kingdom

${ }^{39}$ Department of Physics, University of Regina, Regina, Saskatchewan, Canada

${ }^{40}$ Department of Physics and Astronomy, University of Rochester, Rochester, New York, USA

${ }^{41}$ Department of Physics, Royal Holloway University of London, Egham, Surrey, United Kingdom

${ }^{42}$ III. Physikalisches Institut, RWTH Aachen University, Aachen, Germany

${ }^{43}$ Department of Physics and Astronomy, University of Sheffield, Sheffield, United Kingdom

${ }^{44}$ Institute of Physics, University of Silesia, Katowice, Poland

${ }^{45}$ STFC, Rutherford Appleton Laboratory, Harwell Oxford, United Kingdom and Daresbury Laboratory, Warrington, United Kingdom

${ }^{46}$ Department of Physics, University of Tokyo, Tokyo, Japan

${ }^{47}$ Institute for Cosmic Ray Research, Kamioka Observatory, University of Tokyo, Kamioka, Japan

${ }^{48}$ Institute for Cosmic Ray Research, Research Center for Cosmic Neutrinos, University of Tokyo, Kashiwa, Japan

${ }^{49}$ Department of Physics, Tokyo Metropolitan University, Tokyo, Japan

${ }^{50}$ Department of Physics, University of Toronto, Toronto, Ontario, Canada

${ }^{51}$ TRIUMF, Vancouver, British Columbia, Canada

${ }^{52}$ Department of Physics and Astronomy, University of Victoria, Victoria, British Columbia, Canada

${ }^{53}$ Faculty of Physics, University of Warsaw, Warsaw, Poland

${ }^{54}$ Institute of Radioelectronics, Warsaw University of Technology, Warsaw, Poland

${ }^{55}$ Department of Physics, University of Warwick, Coventry, United Kingdom

${ }^{56}$ Department of Physics, University of Washington, Seattle, Washington, USA

${ }^{57}$ Department of Physics, University of Winnipeg, Winnipeg, Manitoba, Canada

${ }^{58}$ Faculty of Physics and Astronomy, Wroclaw University, Wroclaw, Poland

${ }^{59}$ Faculty of Engineering, Yokohama National University, Yokohama, Japan

${ }^{60}$ Department of Physics and Astronomy, York University, Toronto, Ontario, Canada

(Received 3 January 2017; revised manuscript received 10 March 2017; published 10 April 2017)

$\mathrm{T} 2 \mathrm{~K}$ reports its first results in the search for $C P$ violation in neutrino oscillations using appearance and disappearance channels for neutrino- and antineutrino-mode beams. The data include all runs from January 
2010 to May 2016 and comprise $7.482 \times 10^{20}$ protons on target in neutrino mode, which yielded in the far detector $32 e$-like and $135 \mu$-like events, and $7.471 \times 10^{20}$ protons on target in antineutrino mode, which yielded $4 e$-like and $66 \mu$-like events. Reactor measurements of $\sin ^{2} 2 \theta_{13}$ have been used as an additional constraint. The one-dimensional confidence interval at $90 \%$ for the phase $\delta_{C P}$ spans the range $(-3.13,-0.39)$ for normal mass ordering. The $C P$ conservation hypothesis $\left(\delta_{C P}=0, \pi\right)$ is excluded at $90 \%$ C.L.

DOI: 10.1103/PhysRevLett.118.151801

Introduction.-A new source of $C P$ violation beyond the Cabibbo-Kobayashi-Masakawa quark mixing matrix is necessary to explain observations of baryon asymmetry in the Universe. In the lepton sector the Pontecorvo-MakiNakagawa-Sakata framework $[1,2]$ allows for $C P$ violation. The first indication of nonzero $\theta_{13}$ [3] followed by its discovery [4-6] and then the discovery of $\nu_{\mu} \rightarrow \nu_{e}$ oscillation by T2K [7] have opened the possibility to look for $C P$ violation in neutrino oscillation.

In this Letter we present the first joint fit of neutrino and antineutrino $\stackrel{(-)}{\nu}_{\mu} \rightarrow \stackrel{(-)}{\nu}_{e}$ and $\stackrel{(-)}{\nu}_{\mu} \rightarrow \stackrel{(-)}{\nu}_{\mu}$ oscillation at T2K. The mixing of neutrinos in the three-flavor framework is represented by the unitary PMNS matrix, parameterized by three mixing angles, $\theta_{12}, \theta_{13}$, and $\theta_{23}$, and a $C P$-violating phase $\delta_{C P}$ [8]. The probability for $\stackrel{(-)}{\nu}_{\mu} \rightarrow \stackrel{(-)}{\nu}_{e}$ oscillation, as a function of neutrino propagation distance $L$ and energy $E$, can be written

$$
\begin{aligned}
& P\left(\stackrel{(-)}{\nu}_{\mu} \rightarrow \stackrel{(-)}{\nu}_{e}\right) \\
& \simeq 4 c_{13}^{2} s_{13}^{2} s_{23}^{2} \sin ^{2} \phi_{31}\left[1+\frac{(-)}{\Delta m_{31}^{2}}\left(1-2 s_{13}^{2}\right)\right] \\
& \quad \stackrel{(+)}{-} 8 c_{13}^{2} c_{12} c_{23} s_{12} s_{13} s_{23} \sin \phi_{32} \sin \phi_{31} \sin \phi_{21} \sin \delta_{C P} \\
& \quad \stackrel{( \pm)}{-} 8 c_{13}^{2} s_{13}^{2} s_{23}^{2}\left(1-2 s_{13}^{2}\right) \frac{a L}{4 E} \cos \phi_{32} \sin \phi_{31} \\
& \quad+(C P \text {-even, solar terms }),
\end{aligned}
$$

where $s_{i j}=\sin \theta_{i j}, \quad c_{i j}=\cos \theta_{i j}, \phi_{i j}=\Delta m_{i j}^{2} L / 4 E$, and $\Delta m_{i j}^{2}=m_{i}^{2}-m_{j}^{2} \quad$ represents the neutrino masssquared difference between mass eigenstates $i$ and $j$. Matter effects are included to first order in the terms $a\left[\mathrm{eV}^{2} / c^{4}\right]=7.56 \times 10^{-5} E[\mathrm{GeV}] \rho\left[\mathrm{g} / \mathrm{cm}^{3}\right]$. Our analyses use the complete probability calculation, without approximating matter effects. The $\stackrel{(-)}{\nu}_{\mu} \rightarrow \stackrel{(-)}{\nu}_{\mu}$ survival probability is dominated by the parameters $\sin ^{2} \theta_{23}$ and $\Delta m_{32}^{2}$, as given in [9]. Comparing electron neutrino and antineutrino

Published by the American Physical Society under the terms of the Creative Commons Attribution 4.0 International license. Further distribution of this work must maintain attribution to the author(s) and the published article's title, journal citation, and DOI. appearance probabilities allows a direct measurement of $C P$ violation at $\mathrm{T} 2 \mathrm{~K}$. The asymmetry variable $\left[A_{C P}=\right.$ $\left.P\left(\nu_{\mu} \rightarrow \nu_{e}\right)-P\left(\bar{\nu}_{\mu} \rightarrow \bar{\nu}_{e}\right)\right] /\left[P\left(\nu_{\mu} \rightarrow \nu_{e}\right)+P\left(\bar{\nu}_{\mu} \rightarrow \bar{\nu}_{e}\right)\right]$ and the $\nu_{\mu}\left(\bar{\nu}_{\mu}\right)$ component of the expected T2K flux without oscillations are shown in Fig. 1. At the flux peak energy, $A_{C P}$ can be as large as 0.4 , including a contribution of around 0.1 due to matter effects.

The T2K experiment.-The T2K experiment [10] uses a $30-\mathrm{GeV}$ proton beam from the J-PARC accelerator facility to produce a muon (anti)neutrino beam. The proton beam strikes a graphite target to produce charged pions and kaons, which are focused by three magnetic horns. Depending on the polarity of the horn current, either positively or negatively charged mesons are focused, resulting in a beam largely composed of muon neutrinos or antineutrinos. A 96-m decay volume lies downstream of the magnetic horns, followed by the beam dump and muon monitor [11]. The neutrino beam is measured by detectors placed on axis and off axis at $2.5^{\circ}$ relative to the beam direction. The off-axis neutrino energy spectrum peaks at $0.6 \mathrm{GeV}$, and has a reduced $\stackrel{(-)}{\nu}_{\mu}$ contamination and smaller backgrounds from higher-energy neutrinos than the on-axis spectrum. Two detectors located $280 \mathrm{~m}$ from the target are used to measure the beam direction, spectrum, and composition, as well as

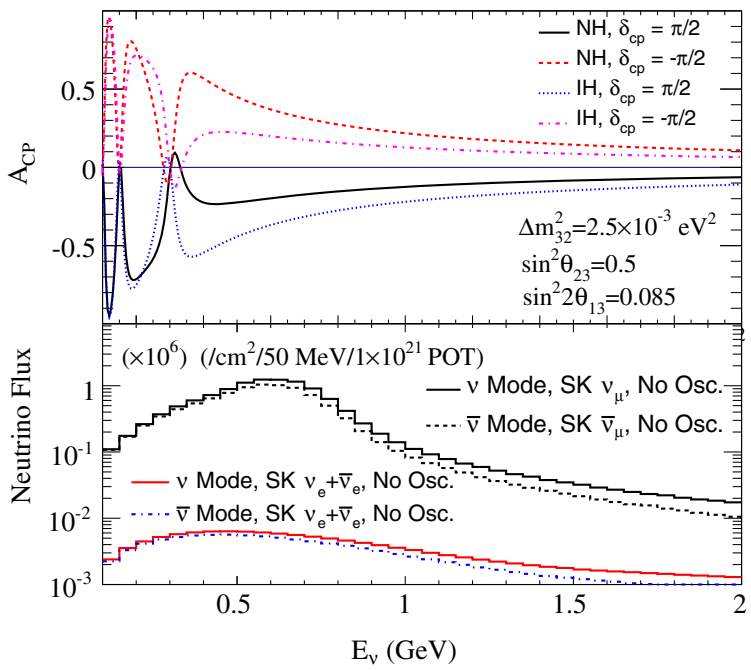

FIG. 1. The leptonic $C P$ asymmetry, $A_{C P}=\left[P\left(\nu_{\mu} \rightarrow \nu_{e}\right)-\right.$ $\left.P\left(\bar{\nu}_{\mu} \rightarrow \bar{\nu}_{e}\right)\right] /\left[P\left(\nu_{\mu} \rightarrow \nu_{e}\right)+P\left(\bar{\nu}_{\mu} \rightarrow \bar{\nu}_{e}\right)\right]$, as a function of energy for maximal $C P$-violation hypotheses (top) and the $\nu_{\mu}\left(\bar{\nu}_{\mu}\right)$ and $\nu_{e}+\bar{\nu}_{e}$ components of the unoscillated neutrino flux in neutrino and antineutrino modes (bottom). 
the event rate: INGRID (on axis) [12] and ND280 (off axis), which is housed inside a 0.2-T magnet. The SuperKamiokande (Super-K) 50-kt water Cherenkov detector [13], located off axis and $295 \mathrm{~km}$ from the neutrino production point, is used to detect oscillated neutrinos.

Data sets. - The results presented here are based on data collected in two periods: one in which the beam operated solely in neutrino mode, January 2010-May 2013, and one in which the beam operated mostly in antineutrino mode, May 2014-May 2016. This comprises a neutrino beam exposure of $7.482 \times 10^{20}$ protons on target (POT) in neutrino mode and $7.471 \times 10^{20}$ POT in antineutrino mode for the far-detector analysis, and an exposure of $5.82 \times 10^{20}$ POT in neutrino mode and $2.84 \times 10^{20}$ POT in antineutrino mode for the near-detector analysis.

Analysis strategy.-The analysis strategy is similar to that of previous T2K results $[7,9,14,15]$ : oscillation parameters are estimated by comparing predictions and observations at the far detector. A tuned prediction of the oscillated spectrum at the far detector, with associated uncertainty, is obtained by fitting samples of charged-current interactions at ND280. The analysis presented here differs from previous results in that both neutrino and antineutrino samples are fitted at both ND280 and Super-K. Including antineutrino data at ND280 ensures that the interaction model is consistent between neutrinos and antineutrinos. Additionally, the use of a magnetized near detector with charge-selected samples in both neutrino and antineutrino beams allows a constraint on wrong-sign contaminations in the beam.

Neutrino flux model.-The T2K neutrino and antineutrino fluxes at near and far detectors, and their correlations, are calculated [16] using a data-driven hybrid simulation with FLUKA 2011 [17] used to simulate hadronic interactions and transport particles inside the target, while GEANT3 [18] with GCALOR [19] is used to simulate the rest of the neutrino beam line. The interactions of hadrons in both FLUKA 2011 and GCALOR are tuned using thin target hadron production data, including measurements of the total cross section for particle production, and $\pi^{ \pm}, K^{ \pm}, p^{+}$, $\Lambda$, and $K_{S}^{0}$ production with $30-\mathrm{GeV}$ protons on a graphite target by the NA61/SHINE experiment [20]. Dominant systematic error sources include uncertainties on the NA61/ SHINE hadron production measurements, hadronic interaction length measurements from NA61/SHINE and other experiments, the initial proton beam trajectory, and the horn currents. The total uncertainty on the flux near the peak energy is $\sim 9 \%$. The $\nu_{\mu}\left(\bar{\nu}_{\mu}\right)$ component of the predicted fluxes without oscillations are shown in Fig. 1. At the far detector and in the absence of oscillations, we predict that $94.1 \%(92.3 \%)$ of the T2K neutrino-mode (antineutrinomode) beam below $1.25 \mathrm{GeV}$ is $\nu_{\mu}\left(\bar{\nu}_{\mu}\right)$. The $\bar{\nu}_{\mu}$ flux in antineutrino mode is reduced by $\sim 20 \%$ relative to the $\nu_{\mu}$ flux in neutrino mode due to the smaller production cross section for $\pi^{-}$relative to $\pi^{+}$in $30 \mathrm{GeV} p+C$ interactions.
Neutrino interaction model.-The interactions of neutrinos and antineutrinos with nuclei in the near and far detectors are modeled with the NEUT [21] neutrino interaction generator. The charged-current quasielastic (CCQE) interactions are modeled with a relativistic Fermi gas nuclear model with relativistic corrections for long-range correlations using the random phase approximation (RPA) as applied by Nieves et al. [22]. The choice of the CCQE nuclear model was made based on fits to external CCQElike data [23] from the MiniBooNE [24,25] and MINERvA $[26,27]$ experiments. Interactions on more than one nucleon are modeled with an implementation of the $2 p-2 h$ model developed by Nieves et al. [28,29]. These interactions are characterized by multinucleon ejection and no final state pions; hence, they may be confused for CCQE interactions in a water Cherenkov detector. The single-pion production model in NEUT has been tuned using form factors from Graczyk and Sobczyk [30] and with a reanalysis of Argonne National Laboratory and Brookhaven National Laboratory bubble chamber data sets [31]. The coherent pion production model has been tuned to reproduce data from MINERvA [32] and T2K [33]. At the T2K peak energy, the antineutrino cross section is $\sim 3.5$ times smaller than the neutrino cross section.

The parameterization of uncertainties in the neutrino interaction model is largely unchanged from previous measurements $[14,15]$. Parameters that vary the binding energy, Fermi momentum, $2 p-2 h$ normalization, and charged current $(\mathrm{CC})$ coherent pion production crosssection normalization are applied separately for interactions on carbon and oxygen. To cover the different predictions by Nieves et al. [28,29] and Martini et al. [34,35] of the relative $2 p$ - $2 h$ interaction rates for neutrinos and antineutrinos, the normalizations of $2 p-2 h$ interactions for neutrinos and antineutrinos are allowed to vary independently.

Only the interactions of $\nu_{\mu}$ and $\bar{\nu}_{\mu}$ are explicitly constrained by near-detector measurements in this analysis. Since the oscillation signals include $\nu_{e}$ and $\bar{\nu}_{e}$ interactions, it is necessary to assign uncertainties on the cross-section ratios $\sigma_{\nu_{e}} / \sigma_{\nu_{\mu}}$ and $\sigma_{\bar{\nu}_{e}} / \sigma_{\bar{\nu}_{\mu}}$. Following the treatment in [36], separate parameters for $\sigma_{\nu_{e}} / \sigma_{\nu_{\mu}}$ and $\sigma_{\bar{\nu}_{e}} / \sigma_{\bar{\nu}_{\mu}}$ are introduced with a theoretical uncertainty of $2.8 \%$ for each. A correlation coefficient of -0.5 is assumed for these two parameters.

Fit to near-detector data.-The systematic parameters in the neutrino flux and interaction models are constrained by a fit to CC candidate samples in the ND280 [10] near detector. The data sets used consist of reconstructed interactions in two fine-grained detectors (FGDs) [37] with particle tracking in three time projection chambers (TPCs) [38]. FGD2 contains six 2.54-cm-thick water panels, allowing systematic parameters governing neutrino interactions on $\mathrm{H}_{2} \mathrm{O}$, the same target as Super-K, to be directly constrained. The CC candidate samples in ND280 are divided into categories based on the beam mode (neutrino vs antineutrino), the FGD in which the interaction takes 
place, the muon charge, and the final-state multiplicity. For data taken in neutrino mode, only interactions with a negatively charged muon are considered. For data taken in antineutrino mode, there are separate categories for events with positively charged (right-sign) and negatively charged (wrong-sign) muon candidates. The wrong-sign candidates are included because the larger neutrino cross section leads to a non-negligible wrong-sign background in antineutrino mode. In neutrino mode, there are three categories for reconstructed final states: no pion candidate in the final state $(\mathrm{CC} 0 \pi)$, one pion candidate in the final state $(\mathrm{CC} 1 \pi)$, and all other $\mathrm{CC}$ candidates (CC other). In antineutrino mode, events are divided into two categories based on the final states: only the muon track exits the FGD to enter the TPC (CC 1-track) and at least one other track enters the TPC (CC $N$-track)

When fitting, the data are binned according to the momentum of the muon candidate, $p_{\mu}$, and $\cos \theta_{\mu}$, where $\theta_{\mu}$ is the angle of the muon direction relative to the central axis of the detector, roughly $1.7^{\circ}$ away from the incident (anti)neutrino direction. A binned maximum likelihood fit is performed in which the neutrino flux and interaction model parameters are allowed to vary. Nuisance parameters describing the systematic errors in the ND280 detector

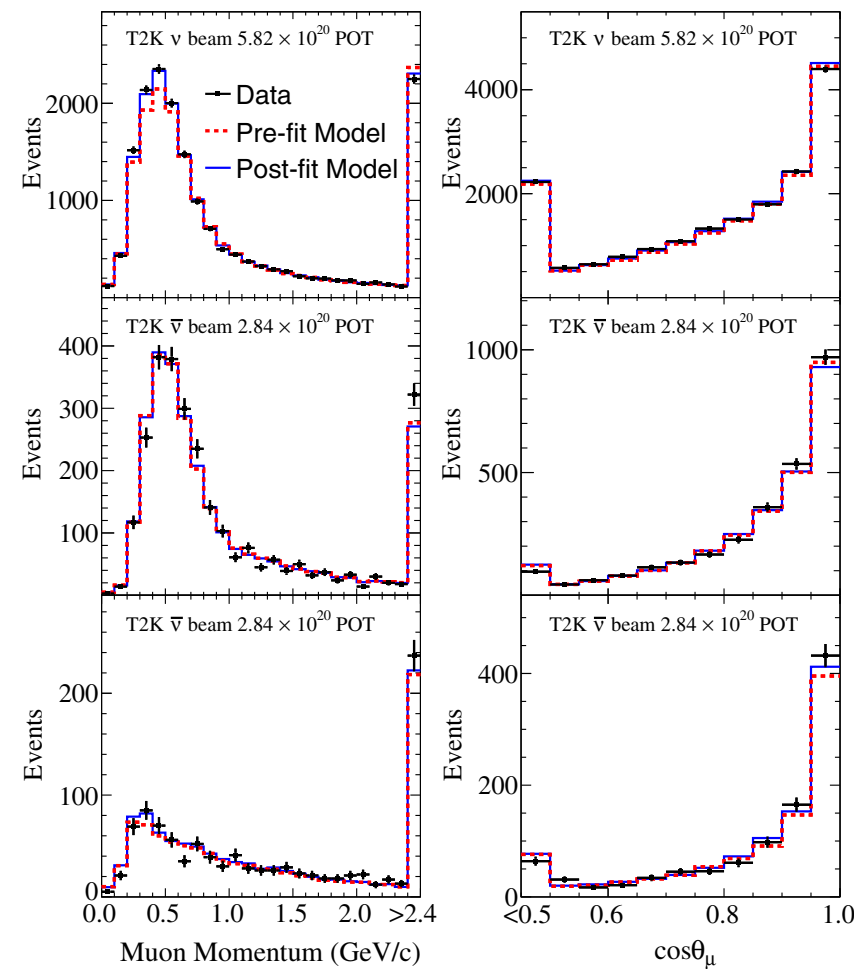

FIG. 2. The FGD2 data, prefit predictions and postfit predictions binned in $p_{\mu}$ (left) and $\cos \theta_{\mu}$ (right) for the neutrino mode $\mathrm{CC} 0 \pi$ (top), antineutrino mode CC 1 -track $\mu^{+}$(middle) and antineutrino mode CC 1-track $\mu^{-}$(bottom) categories. The overflow bins are integrated out to $10000 \mathrm{MeV} / c$ for $p_{\mu}$ and -1.0 for $\cos \theta_{\mu}$ respectively. model - the largest of which is pion interaction modelingare marginalized in the fit.

The fitted $p_{\mu}$ and $\cos \theta_{\mu}$ distributions for the FGD2 $\mathrm{CC} 0 \pi$ and $\mathrm{CC}$ 1-track categories are shown in Fig. 2. Acceptable agreement between the postfit model and data is observed for both kinematic variables, with a $p$ value of 0.086 . The best-fit fluxes are increased with respect to the original flux model by $10 \%-15 \%$ near the flux peak. This is driven by the prefit deficit in the prediction for the $\mathrm{CC} 0 \pi$ and $\mathrm{CC}$ other samples. The fitted value for the axial mass in the CCQE model is $1.12 \mathrm{GeV} / c^{2}$, compared to $1.24 \mathrm{GeV} / c^{2}$ in a previous fit where the $2 p-2 h$ model and RPA corrections were not included [14]. The lower axial mass decreases the interaction rate, driving the increased flux prediction. The fit to ND280 data reduces the uncertainty on the event-rate predictions at the far detector due to uncertainties on the flux and ND280-constrained interaction model parameters from $10.9 \%(12.4 \%)$ to $2.9 \%$ (3.2\%) for the $\nu_{e}\left(\bar{\nu}_{e}\right)$ candidate sample.

Far-detector data.-At the far detector, events are extracted that lie within $[-2,10] \mu$ s relative to the beam arrival. Fully contained events within the fiducial volume are selected by requiring that no hit cluster is observed in the outer detector volume, that the distance from the reconstructed vertex to the inner detector wall is larger than $2 \mathrm{~m}$, and that the total observed charge is greater than the equivalent quantity for a $30-\mathrm{MeV}$ electron. The CCQE component of our sample is enhanced by selecting events with a single Cherenkov ring. The $\nu_{\mu} / \bar{\nu}_{\mu} \mathrm{CCQE}$ candidate samples are then selected by requiring a $\mu$-like ring using a particle identification likelihood, zero or one decay electron candidates, and muon momentum greater than $200 \mathrm{MeV} / c$ to reduce pion background. Post selection, 135 and 66 events remain in the $\nu_{\mu}$ and $\bar{\nu}_{\mu}$ candidate samples, respectively, while if $\left|\Delta m_{32}^{2}\right|=2.509 \times 10^{-3} \mathrm{eV}^{2} / c^{4}$ and $\sin ^{2} \theta_{23}=0.528$ (i.e., maximal disappearance), 135.5 and 64.1 events are expected. The $\nu_{e} / \bar{\nu}_{e}$ CCQE candidate samples are selected by requiring an $e$-like ring and zero decay electron candidates, not $\pi^{0}$-like and reconstructed energy less than $1.25 \mathrm{GeV}$. The total number of events remaining in these samples is presented in Table I with their respective expectation for different values of $\delta_{C P}$, $\sin ^{2} 2 \theta_{13}=0.085, \quad\left|\Delta m_{32}^{2}\right|=2.509 \times 10^{-3} \mathrm{eV}^{2} / c^{4}$, and

TABLE I. Number of $\nu_{e}$ and $\bar{\nu}_{e}$ events expected for various values of $\delta_{C P}$ and both mass orderings compared to the observed numbers.

\begin{tabular}{lccccc}
\hline \hline Normal & $\delta_{C P}=-\pi / 2$ & $\delta_{C P}=0$ & $\delta_{C P}=\pi / 2$ & $\delta_{C P}=\pi$ & Observed \\
\hline$\nu_{e}$ & 28.7 & 24.2 & 19.6 & 24.1 & 32 \\
$\bar{\nu}_{e}$ & 6.0 & 6.9 & 7.7 & 6.8 & 4 \\
Inverted & $\delta_{C P}=-\pi / 2$ & $\delta_{C P}=0$ & $\delta_{C P}=\pi / 2$ & $\delta_{C P}=\pi$ & Observed \\
$\nu_{e}$ & 25.4 & 21.3 & 17.1 & 21.3 & 32 \\
$\bar{\nu}_{e}$ & 6.5 & 7.4 & 8.4 & 7.4 & 4 \\
\hline \hline
\end{tabular}



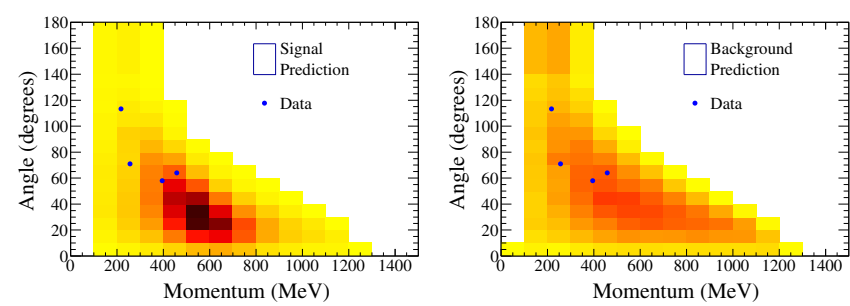

FIG. 3. The reconstructed lepton momentum and angle relative to the beam at the far detector for the $\bar{\nu}_{e}$ sample signal (left) and background (right) expectation with the data overlaid (blue points).
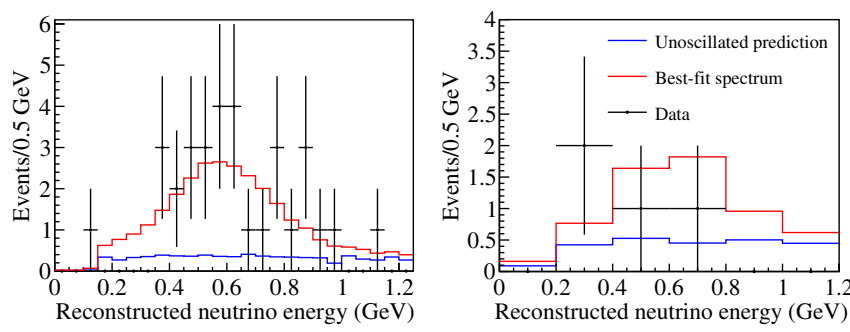

FIG. 4. The reconstructed neutrino energy at the far detector for the $\nu_{e}$ (left) and $\bar{\nu}_{e}$ (right) candidate samples is shown together with the expected distribution without oscillation (blue histogram) and the best fit (red histogram).

$\sin ^{2} \theta_{23}=0.528$. The $\nu_{e}\left(\bar{\nu}_{e}\right)$ contamination in the $\bar{\nu}_{e}\left(\nu_{e}\right)$ sample is $17.4 \%(0.5 \%)$, and the proportion of the sample expected to correspond to oscillated $\bar{\nu}_{e}\left(\nu_{e}\right)$ events is $46.4 \%$ $(80.9 \%)$ for $\delta_{C P}=-\pi / 2$. A more detailed description of the candidate event selections can be found in previous publications [14]. The $\bar{\nu}_{e}$ signal events are concentrated in the forward direction with respect to the beam, unlike the backgrounds (Fig. 3). Therefore, incorporating reconstructed lepton angle information in the analysis increases the sensitivity. The reconstructed neutrino energy spectra for the $\nu_{e}$ and $\bar{\nu}_{e}$ samples is shown in Fig. 4.

The systematic errors concerning the detector behavior are estimated using atmospheric neutrino and cosmic-ray muon events. A sample of hybrid data-Monte Carlo events is also used to evaluate uncertainties regarding $\pi^{0}$ rejection.

TABLE II. Systematic uncertainty on the predicted event rate at the far detector.

\begin{tabular}{lcccc}
\hline \hline Source (\%) & $\nu_{\mu}$ & $\nu_{e}$ & $\bar{\nu}_{\mu}$ & $\bar{\nu}_{e}$ \\
\hline $\begin{array}{l}\text { ND280-unconstrained } \\
\quad \text { cross section }\end{array}$ & 0.7 & 3.0 & 0.8 & 3.3 \\
$\quad \begin{array}{lccc}\text { Flux and ND280-constrained } \\
\quad \text { cross section }\end{array}$ & 2.8 & 2.9 & 3.3 & 3.2 \\
$\quad \begin{array}{l}\text { Super-Kamiokande detector systematics } \\
\quad \text { Final or secondary }\end{array}$ & 3.9 & 2.4 & 3.3 & 3.1 \\
$\quad$ hadron interactions & 1.5 & 2.5 & 2.1 & 2.5 \\
$\quad$ Total & & & & \\
\hline \hline
\end{tabular}

Correlations between the uncertainties for the four samples are taken into account in the fits.

The fractional variation of the number of expected events for the four samples owing to the various sources of systematic uncertainty are shown in Table II. A more indepth description of the sources of systematic uncertainty in the fit is given in [14], although this reference does not cover the updates discussed in previous sections.

Oscillation analysis.-The oscillation parameters $\sin ^{2} \theta_{23}, \Delta m_{32}^{2}, \sin ^{2} \theta_{13}$, and $\delta_{C P}$ are estimated by performing a joint maximum-likelihood fit of the four far-detector samples. The oscillation probabilities are calculated using the full three-flavor oscillation formulas [39]. Matter effects are included with an Earth density of $\rho=2.6 \mathrm{~g} / \mathrm{cm}^{3}$ [40].

As described previously, the priors for the beam flux and neutrino interaction cross-section parameters are obtained from the fit with the near-detector data. The priors [8] for the solar neutrino oscillation parameters-whose impact is almost negligible-are $\sin ^{2} 2 \theta_{12}=0.846 \pm 0.021, \Delta m_{21}^{2}=$ $(7.53 \pm 0.18) \times 10^{-5} \mathrm{eV}^{2} / c^{4}$, and in some fits we use $\sin ^{2} 2 \theta_{13}=0.085 \pm 0.005$ [8], called the "reactor measurement." Flat priors are used for $\sin ^{2} \theta_{23}, \Delta m_{32}^{2}$, and $\delta_{C P}$.

We use a procedure analogous to [15]: we integrate the likelihood over the prior probability density function of the nuisance parameters and we obtain the marginal likelihood which depends only on the relevant oscillation parameters. We define $-2 \Delta \ln \mathcal{L}=-2 \ln \left[\mathcal{L}(\mathbf{0}) / \mathcal{L}_{\max }\right]$ as the ratio between the marginal likelihood at the point $\mathbf{o}$ of the relevant oscillation parameter space and the maximum marginal likelihood.

We have conducted three analyses using different far-detector event quantities and different statistical approaches. All of them use the neutrino energy reconstructed in the CCQE hypothesis $\left(E_{\text {rec }}\right)$ for the $\stackrel{(-)}{\nu}_{\mu}$ samples. The first analysis uses $E_{\text {rec }}$ and the reconstructed angle between the lepton and the neutrino beam direction, $\theta_{\text {lep }}$, of the $\stackrel{(-)}{\nu}$ candidate samples and provides confidence intervals using a hybrid Bayesian-frequentist approach [41]. These results are shown in the following figures. The second analysis is fully Bayesian and uses the lepton momentum, $p_{\text {lep }}$, and $\theta_{\text {lep }}$ for the $\stackrel{(-)}{\nu}_{e}$ samples to compute credible intervals using the posterior probability. The third analysis uses only $E_{\text {rec }}$ spectra for the $\stackrel{(-)}{\nu}_{e}$ samples and a Markov chain Monte Carlo method [42] to provide Bayesian credible intervals. This analysis performs a simultaneous fit of both the near- and far-detector data, providing a validation of the extrapolation of the flux, cross section, and detector systematic parameters from the near to far detector. All three methods are in good agreement.

An indication of the sensitivity to $\delta_{C P}$ and the mass ordering can be obtained from Table I. If $C P$ violation is maximal $\left(\delta_{C P}= \pm \pi / 2\right.$ ), the predicted variation of the total number of events with respect to the $C P$ conservation 


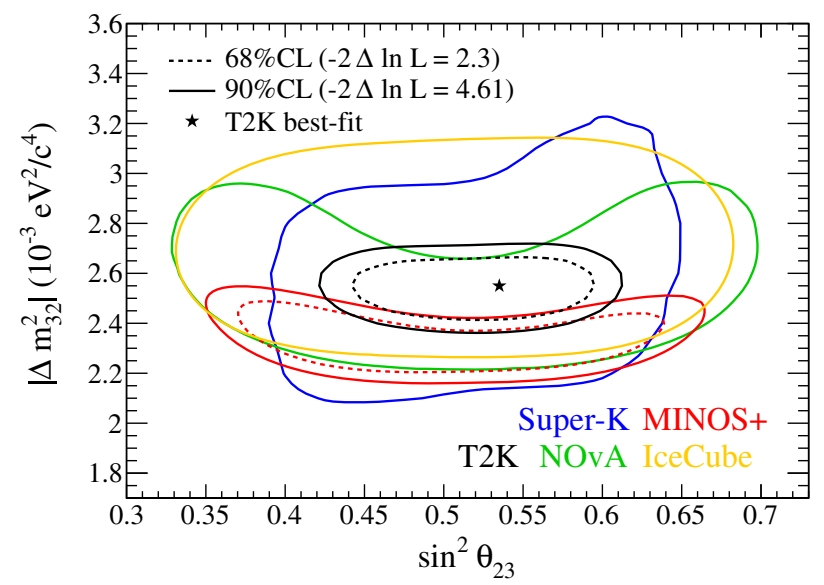

FIG. 5. The $68 \%(90 \%)$ constant $-2 \Delta \ln L$ confidence regions for the $\sin ^{2} \theta_{23}-\left|\Delta m_{32}^{2}\right|$ plane assuming normal ordering, alongside NO $\nu$ A [43], MINOS+[44], SK [45], and IceCube [46] confidence regions.

hypothesis $\left(\delta_{C P}=0, \pi\right)$ is about $20 \%$. The different mass orderings induce a variation of the number of expected events of about $10 \%$. Matter effects are negligible for the $\nu_{\mu}$ and $\bar{\nu}_{\mu}$ candidate samples, while they affect the number of events in the $\nu_{e}$ and $\bar{\nu}_{e}$ candidate samples by about $6 \%$ and $4 \%$, respectively, for maximal $C P$ violation.

A series of fits are performed where one or two oscillation parameters are determined and the others are marginalized. Confidence regions are set using the constant $-2 \Delta \ln L$ method [8]. In the first fit confidence regions in the $\sin ^{2} \theta_{23}-\left|\Delta m_{32}^{2}\right|$ plane (Fig. 5) were computed using the reactor measurement of $\sin ^{2} \theta_{13}$. The best-fit values are $\sin ^{2} \theta_{23}=0.532$ and $\left|\Delta m_{32}^{2}\right|=2.545 \times 10^{-3} \mathrm{eV}^{2} / c^{4}$ $\left(\sin ^{2} \theta_{23}=0.534\right.$ and $\left.\left|\Delta m_{32}^{2}\right|=2.510 \times 10^{-3} \mathrm{eV}^{2} / c^{4}\right)$ for

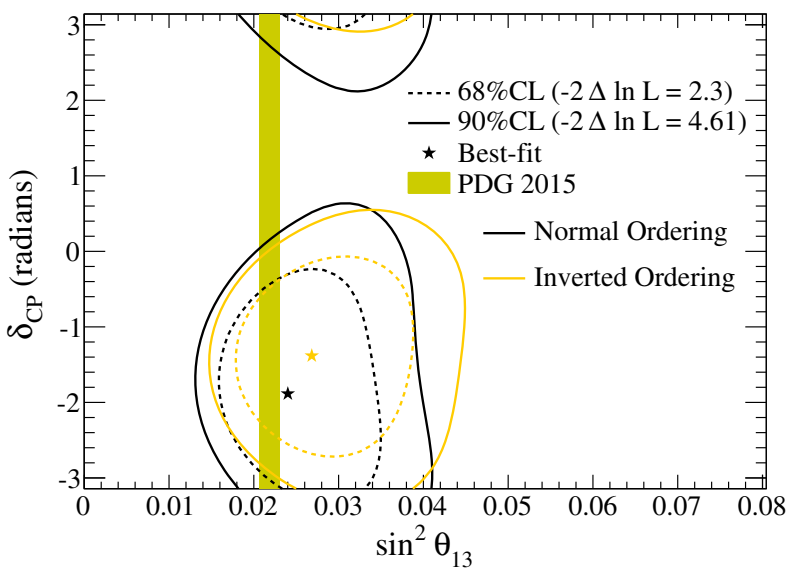

FIG. 6. The $68 \%$ (90\%) constant $-2 \Delta \ln L$ confidence regions in the $\delta_{C P}-\sin ^{2} \theta_{13}$ plane are shown by the dashed (continuous) lines, computed independently for the normal (black) and inverted (yellow) mass ordering. The best-fit point is shown by a star for each mass-ordering hypothesis. The $68 \%$ confidence region from reactor experiments on $\sin ^{2} \theta_{13}$ is shown by the yellow vertical band.

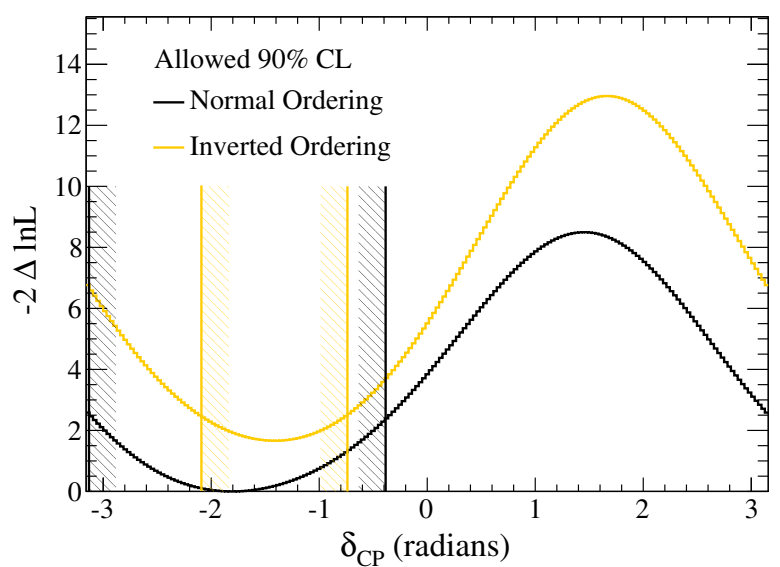

FIG. 7. $-2 \Delta \ln \mathcal{L}$ as a function of $\delta_{C P}$ for the normal (black) and inverted (yellow) mass ordering. The vertical lines show the corresponding allowed $90 \%$ confidence intervals, calculated using the Feldman-Cousins method. $\sin ^{2} \theta_{13}$ is marginalized using the reactor measurement as prior probability.

the normal (inverted) ordering. The goodness of fit for all three analyses is better than $80 \%$. The result is consistent with maximal disappearance. The $\mathrm{T} 2 \mathrm{~K}$ data weakly prefer the second octant $\left(\sin ^{2} \theta_{23}>0.5\right)$ with a posterior probability of $61 \%$.

Confidence regions in the $\sin ^{2} \theta_{13}-\delta_{C P}$ plane are computed independently for both mass-ordering hypotheses (Fig. 6) without using the reactor measurement. The addition of antineutrino samples at Super-K gives the first sensitivity to $\delta_{C P}$ from $\mathrm{T} 2 \mathrm{~K}$ data alone. There is good agreement between the $\mathrm{T} 2 \mathrm{~K}$ result and the reactor measurement for $\sin ^{2} \theta_{13}$. For both mass-ordering hypotheses, the best-fit value of $\delta_{C P}$ is close to $-\pi / 2$.

Confidence intervals for $\delta_{C P}$ are obtained using the Feldman-Cousins method [47]. The parameter $\sin ^{2} \theta_{13}$ is marginalized using the reactor measurement. The best-fit value is obtained for the normal ordering and $\delta_{C P}=-1.791$, close to maximal $C \mathrm{P}$ violation (Fig. 7). For inverted ordering the best-fit value of $\delta_{C P}$ is -1.414 . The hypothesis of $C P$ conservation $\left(\delta_{C P}=0, \pi\right)$ is excluded at $90 \%$ C.L. and $\delta_{C P}=0$ is excluded at more than $2 \sigma$. The $\delta_{C P}$ confidence intervals at $90 \%$ C.L. are $(-3.13,-0.39)$ for normal ordering and $(-2.09,-0.74)$ for inverted ordering. The Bayesian credible interval at $90 \%$, marginalizing over the mass ordering, is $(-3.13,-0.21)$. The normal ordering is weakly favored over the inverted ordering with a posterior probability of $75 \%$.

Sensitivity studies show that, if the true value of $\delta_{C P}$ is $-\pi / 2$ and the mass ordering is normal, the fraction of pseudoexperiments where $C P$ conservation $\left(\delta_{C P}=0, \pi\right)$ is excluded with a significance of $90 \%$ C.L. is $17.3 \%$, with the amount of data used in this analysis.

Conclusions. - T2K has performed the first search for $C P$ violation in neutrino oscillations using $\nu_{\mu} \rightarrow \nu_{e}$ appearance and $\nu_{\mu} \rightarrow \nu_{\mu}$ disappearance channels in neutrino and 
antineutrino modes. The one-dimensional confidence interval at $90 \%$ for $\delta_{C P}$ spans the range $(-3.13,-0.39)$ in the normal mass ordering. The $C P$-conservation hypothesis $\left(\delta_{C P}=0, \pi\right)$ is excluded at $90 \%$ C.L. The data related to the measurements and results presented in this Letter can be found in Ref. [48].

We thank the Japan Proton Accelerator Research Complex (J-PARC) staff for superb accelerator performance. We thank the Conseil Européen pour la Recherche Nucléaire (CERN) North Area experiment 61 (NA61)/SHINE Collaboration for providing valuable particle production data. We acknowledge the support of Ministry of Education, Culture, Sports, Science and Technology (MEXT), Japan; Natural Sciences and Engineering Research Council (NSERC) (Grant No. SAPPJ-2014-00031), National Research Council (NRC) and Canada Foundation for Innovation (CFI), Canada; Commissariat à l'Energie Atomique et aux Energies Alternatives (CEA) and Centre National de la Recherche Scientifique-Institut National de Physique Nucléaire et de Physique des Particules (CNRS/IN2P3), France; Deutsche Forschungsgemeinschaft (DFG), Germany; Istituto Nazionale di Fisica Nucleare (INFN), Italy; National Science Centre $(\mathrm{NCN})$ and Ministry of Science and Higher Education, Poland; Russian Science Foundation (RSF), Russian Foundation for Basic Research (RFBR), and Ministry of Education and Science (MES), Russia; Ministerio de Economía y Competitividad (MINECO) and European Regional Development Fund (ERDF) funds, Spain; Swiss National Science Foundation (SNSF) and State Secretariat for Education, Research and Innovation (SERI), Switzerland; Science and Technology Facilities Council (STFC), UK; and Department of Energy (DOE), USA. We also thank CERN for the Underground Area experiment 1 (UA1)/NOMAD magnet, Deutsches Elektronen-Synchrotron (DESY) for the Hadron-ElektronRing-Anlage-B (HERA-B) magnet mover system, National Insitutue of Informatics (NII) for Science Information Network 4 (SINET4), the Western Research Grid (WestGrid) and SciNet consortia in Compute Canada, and Grid for Particle Physics (GridPP) in the United Kingdom. In addition, participation of individual researchers and institutions has been further supported by funds from European Research Council (ERC) (FP7), H2020 Grant No. RISE-GA644294-JENNIFER, EU; Japan Society for the Promotion of Science (JSPS), Japan; Royal Society, UK; the Alfred P. Sloan Foundation and the DOE Early Career program, USA.

Note added.-Recently, a paper by the $\mathrm{NO} \nu \mathrm{A}$ Collaboration has appeared [49], in which $\sin ^{2} \theta_{23}=0.5$ is disfavored by the data at $2.6 \sigma$. Considering their measurement, $\sin ^{2} \theta_{23}=0.404_{-0.022}^{+0.030}$ for normal ordering, and the fact that the $68 \%$ C.L. interval for T2K measurement [50] extends from 0.464 to 0.578 , with a best fit of 0.532 , the tension between the two measurements is rather mild $(1.7 \sigma)$. Several systematic effects (including additional smearing effects on the reconstructed energy) might produce a bias in the $\sin ^{2} \theta_{23}$ measurement and they must be studied with care. We have investigated them, including possible multinucleon knockout in neutrino-nucleus interactions [15]. This last effect is not a significant uncertainty source at the present statistical precision.

*Also at J-PARC, Tokai, Japan.

${ }^{\dagger}$ Also at Kavli IPMU (WPI), University of Tokyo, Tokyo, Japan.

\#Also at National Research Nuclear University "MEPhI" and Moscow Institute of Physics and Technology, Moscow, Russia.

${ }^{\S}$ Also at JINR, Dubna, Russia.

"Also at Science Department, BMCC/CUNY, New York, New York, USA.

"Also at Institute of Particle Physics, Canada.

[1] Z. Maki, M. Nakagawa, and S. Sakata, Prog. Theor. Phys. 28, 870 (1962).

[2] B. Pontecorvo, Sov. Phys. JETP 26, 984 (1968).

[3] K. Abe et al. (T2K Collaboration), Phys. Rev. Lett. 107, 041801 (2011).

[4] F. P. An et al., Phys. Rev. Lett. 108, 171803 (2012).

[5] J. K. Ahn et al., Phys. Rev. Lett. 108, 191802 (2012).

[6] Y. Abe et al. (Double Chooz Collaboration), Phys. Rev. Lett. 108, 131801 (2012).

[7] K. Abe et al. (T2K Collaboration), Phys. Rev. Lett. 112, 061802 (2014).

[8] K. A. Olive et al. (Particle Data Group Collaboration), Chin. Phys. C 38, 090001 (2014); and http://pdg.lbl.gov/2015/.

[9] K. Abe et al. (T2K Collaboration), Phys. Rev. Lett. 111, 211803 (2013).

[10] K. Abe et al. (T2K Collaboration), Nucl. Instrum. Methods Phys. Res., Sect. A 659, 106 (2011).

[11] K. Suzuki et al. (T2K Collaboration), Prog. Theor. Exp. Phys. (2015) 053C01.

[12] K. Abe et al., Nucl. Instrum. Methods Phys. Res., Sect. A 694, 211 (2012).

[13] Y. Fukuda et al., Nucl. Instrum. Methods Phys. Res., Sect. A 501, 418 (2003).

[14] K. Abe et al. (T2K Collaboration), Phys. Rev. D 91, 072010 (2015).

[15] K. Abe et al. (T2K Collaboration), Phys. Rev. Lett. 116, 181801 (2016).

[16] K. Abe et al. (T2K Collaboration), Phys. Rev. D 87, 012001 (2013); 87, 019902 (2013).

[17] A. Ferrari, P. R. Sala, A. Fasso, and J. Ranft, Reports No. CERN-2005-010, SLAC-R-773. and INFN-TC-05$11,2005$.

[18] R. Brun, F. Bruyant, F. Carminati, S. Giani, M. Maire, A. McPherson, G. Patrick, and L. Urban, Report No. CERNW5013, CERN, 1994.

[19] C. Zeitnitz and T. A. Gabriel, Nucl. Instrum. Methods Phys. Res., Sect. A 349, 106 (1994). 
[20] N. Abgrall et al. (NA61/SHINE Collaboration), Eur. Phys. J. C 76, 84 (2016).

[21] Y. Hayato, Acta Phys. Pol. B 40, 2477 (2009). Version 5.3.2 of NEUT library is used, which includes (i) the multinucleon ejection model of Nieves et al. [23] and (ii) nuclear longrange correlations for $\mathrm{CCQE}$ interactions, treated in the random phase approximation [24].

[22] J. Nieves, J. E. Amaro, and M. Valverde, Phys. Rev. C 70, 055503 (2004); 72, 019902(E) (2005).

[23] C. Wilkinson et al., Phys. Rev. D 93, 072010 (2016).

[24] A. A. Aguilar-Arevalo et al. (MiniBooNE Collaboration), Phys. Rev. D 81, 092005 (2010).

[25] A. A. Aguilar-Arevalo et al. (MiniBooNE Collaboration), Phys. Rev. D 88, 032001 (2013).

[26] G. Fiorentini et al. (MINERvA Collaboration), Phys. Rev. Lett. 111, 022502 (2013).

[27] L. Fields et al. (MINERvA Collaboration), Phys. Rev. Lett. 111, 022501 (2013).

[28] J. Nieves, I. R. Simo, and M. J. Vicente Vacas, Phys. Rev. C 83, 045501 (2011).

[29] R. Gran, J. Nieves, F. Sanchez, and M. J. Vicente Vacas, Phys. Rev. D 88, 113007 (2013).

[30] K. M. Graczyk and J. T. Sobczyk, Phys. Rev. D 77, 053001 (2008); 79, 079903(E) (2009).

[31] C. Wilkinson, P. Rodrigues, S. Cartwright, L. Thompson, and K. McFarland, Phys. Rev. D 90, 112017 (2014).

[32] A. Higuera et al. (MINERvA Collaboration), Phys. Rev. Lett. 113, 261802 (2014).

[33] K. Abe et al. (T2K Collaboration), Phys. Rev. Lett. 117, 192501 (2016).

[34] M. Martini, M. Ericson, G. Chanfray, and J. Marteau, Phys. Rev. C 80, 065501 (2009).
[35] M. Martini and M. Ericson, Phys. Rev. C 87, 065501 (2013).

[36] M. Day and K. S. McFarland, Phys. Rev. D 86, 053003 (2012).

[37] P. Amaudruz et al. (T2K ND280 FGD Collaboration), Nucl. Instrum. Methods Phys. Res., Sect. A 696, 1 (2012).

[38] N. Abgrall et al. (T2K ND280 TPC Collaboration), Nucl. Instrum. Methods Phys. Res., Sect. A 637, 25 (2011).

[39] V. D. Barger, K. Whisnant, S. Pakvasa, and R. J. N. Phillips, Phys. Rev. D 22, 2718 (1980).

[40] K. Hagiwara, N. Okamura, and K.-i. Senda, J. High Energy Phys. 09 (2011) 082.

[41] R. D. Cousins and V. L. Highland, Nucl. Instrum. Methods Phys. Res., Sect. A 320, 331 (1992).

[42] W. K. Hastings, Biometrika 57, 97 (1970).

[43] P. Adamson et al. (NO $\nu$ A Collaboration), Phys. Rev. D 93, 051104 (2016).

[44] P. Adamson et al., Phys. Rev. Lett. 25, 181801 (2013).

[45] R. Wendell, Proc. Sci., ICRC2015 (2015) 1062.

[46] M. Aartsen et al., Nucl. Phys. B908, 161 (2016).

[47] G. J. Feldman and R. D. Cousins, Phys. Rev. D 57, 3873 (1998).

[48] K. Abe et al. (T2K Collaboration), http://t2k-experiment .org/results/t2kdata-nu-antinu-joint-analysis-2016.

[49] L. Adamson et al. ( $\mathrm{NO} \nu \mathrm{A}$ Collaboration), following Letter, Phys. Rev. Lett. 118, 151802 (2017).

[50] See Supplemental Material at http://link.aps.org/ supplemental/10.1103/PhysRevLett.118.151801 for the figures of the reconstructed energy for the far-detector muon neutrino candidate samples together with the best fit. 